Biotechnology Research

http:/www.journals.zu.edu.eg/journalDisplay.aspx?Journalld=1\&queryType=Master

\title{
GENETIC EVALUATION OF EARLINESS AND SPINELESS SAFFLOWER MUTANT LINES FOR QUANTITATIVE AND OIL CONTENT CRITERIA
}

\author{
Marwa E. Ahmed", S.S. Soliman, M.K. Amin and M.A.H. Youssef \\ Genet. Dept., Fac. Agric., Zagazig Univ., Egypt
}

Received: 04/09/2019 ; Accepted: 20/10/2019

\begin{abstract}
This investigation was carried out at experimental and research Farm, Faculty of Agriculture, Zagazig University, Egypt during six winter seasons 2012/2013, 2013/2014, 2014/2015, $2015 / 2016,2016 / 2017$ and 2017/2018. The aim of this study is to evaluate earliness and spineless mutant lines of safflower. The morphological traits (earliness, number of days to maturity) were recorded for two lines of safflower, line III and line VI and their promising mutant lines. The results confirmed the stability of 8 promising mutant lines for line III, $(4,7,9,10,12,14,17,18)$ and 6 promising mutant lines for line VI, $(1,2,5,6,9,7)$. In the M10 generation, 9 criteria were recorded, 1plant height $(\mathrm{cm}), 2$-number of branches per plant, 3-number of capitula per plant, 4-number of seeds per capitulam,5-number of seeds per plant, 6-100 seed weight (g), 7-seed weight/plant (g), 8-days to maturity, 9- oil (\%). High broad sense heritability $\left(\mathrm{h}^{2}\right)$ of No. of capitula per plant, 100-seed weight, seed weight per plant, days to maturity and oil content were $79.16 \%, 77.95 \%, 94.91 \%, 79.23 \%$ and $69.41 \%$, respectively among line III and their mutant lines. The present study confirmed that the relationship between characters under study varied between genotypes for example, oil content slightly negative correlated with seed weight per plant and was different among genotypes $(-0.2495$, 0.0678 and -0.2358 ) for line III and line VI and for over all genotypes, respectively. These results confirmed that selection of oil content and seed weight per plant could be achieved in line VI and their mutants (- 0.0678). In addition, highly positive correlation between seed weight per plant and each of No. of capitula per plant and No. of seeds per plant at line VI, was observed. These results showed the simple heritable system of days to maturity, than the spineless criteria. These promising mutant lines had 168 and 170 days to maturity of line 4 and 7 from line III and 177 and 182 days of line 2 and 5 from line VI by comparison to 180 and 188 days for control of line III and line VI, respectively. These results suggest the improvement possibility of new Egyptian varieties for cultivation of harsh and poor land desert.
\end{abstract}

Key words: Safflower (Carthamus tinctorius L.), earliness, spineless, quantitative, heritability $\left(\mathrm{h}^{2}\right)$, oil content, correlation coefficients (r).

\section{INTRODUCTION}

Safflower (Carthamus tinctorius L.) $2 \mathrm{n}=24$ chromosomes, family Asteraceae is one of the oldest domesticated crops. It has been grown since ancient times both as a dye and as an oil crop in a wide range of geographical regions (Knowles, 1976). (Weiss, 1971) reported that safflower has been recorded as being grown for centuries in a wide area covering southern and western China, much of India and westward

\footnotetext{
*Corresponding author: Tel. : +201007010230

E-mail address: marwaelashmoy@yahoo.com
}

across present-day Pakistan, Afghanistan, Iran, Iraq, northern Saudia Arabia, Kazakhstan, Turkey, and numerous other middle eastern countries, as well down Nile valley of Egypt, Sudan, and Ethiopia.

The western expansion of the arabs in creating the muslim empire of the $5^{\text {th }}$ and $6^{\text {th }}$ centuries probably helped the cultivation of safflower along the Maghreb and into Europe. Safflower seeds have been found in 4,000 yearold Egyptian tombs and their use were recorded 
in China approximately 2,200 years ago. The flowers of the safflower plant have long been used as a source of yellow and red dyes for clothing and food. The petals have also been used for medical purposes and as a stimulant for blood circulation and phlegm reduction, and for healing fractures, contusions, and strains (Smith and Jimmerson, 2005).

Three principle products come from current safflower production: oil, meal, and birdseed. Oil is the primary product and has food and industrial uses. There are two types of safflower oil. The first oil is high in monounsaturated fatty acid (oleic) and the second is high in polyunsaturated fatty acid (linoleic). Oleic oils are used as cooking oils. Linoleic oil is used as a drying agent in paints and varnishes because linoleic oil does not yellow (Smith and Jimmerson, 2005).

Safflower is usually considered to be a selfpollinated crop. However, out-crossing between safflower crops has been reported to be anywhere from 0 to $100 \%$ (Claassen, 1950), Characteristics that have been used to measure out-crossing include allozymes, flower color, spiny versus non-spiny, dominant white seed hull versus recessive gray strip and high linoleic/ low oleic versus low linoleic/high oleic fatty acid content. High-oleic safflower oil is lower in saturates and higher in monounsaturates than olive oil and is beneficial in preventing coronary artery diseases and tend to lower blood levels of low density level (LDL) (bad cholesterol) without affecting high density level (HDL) (good cholesterol) (Zhaomu and Lijie, 2001).

In addition, safflower (Carthamus tinctorius L.) nowadays has gained the reputation of being an edible oil of superior quality containing high levels of unsaturated fatty acids, such as oleic and linoleic acids, associated with the reduction of cholesterol level in the human blood (Chaturvedi et al., 2001). It is also a source of important biochemicals like tocopherol in oil and carthamin in flowers (Ramaswamy, 2001). Safflower has a deep root system allowing the plant to utilize efficiently the nutrients that may not be available to small-grain crops. Hence, introduction of such crop will enhance the sustainability of the organic farming system and benefits consumers and farmers. Therefore the safflower has been introduced in organic farming in Central Europe.

Moreover, safflower can be grown successfully on soil with poor fertility and in areas with relatively low temperatures, safflower also classified as a moderately salt-tolerant plant (Siddiqi et al., 2007). World production of safflower has decreased as the crop suffered from increased cultivation of sunflower, soya and rape (Weiss, 2000). The estimated world production is about 0.622 million tons of seed per year from about 0.736 million hectares (FAO, 2009). At recent statistics according to FAO (2014) statistics, safflower production in the world was realized on an area of $1,010,180$ ha with a total world production reaching about 867,659 tons (Yilmaz et al., 2016).

Conventional breeding has not been effective in boosting the per-hectare yields of either "oil" or "seed". Genetic upgradation of the "ultimate product" in safflower is complicated, as it involves simultaneous improvement of seed yield and oil content. Such problems may alternatively be resolved by "Mutagenesis", where sufficient genetic variability for characters under consideration can be created. (Khadeer and Anwar, 1991) used large mutagens $(\gamma$-ray, EMS, NMU, and sodium azide SA). The results thus obtained, suggest that for a complex trait like oil quality and/or quantity, sufficient variability can be induced by mutagenesis besides polygenic traits and induced variability can be exploited by the breeder for the genetic improvement of desirable traits in safflower. Multiple investigations were done for induction of genetic variability by using mutagenesis (Veena and Ravikumar, 2003; Velasco et al., 2005; Mozaffari and Asadi, 2006).

In Egypt, safflower area decreased year after year at upper Egypt, because the local genotypes suffering many problems as lateness (180-190 days at maturity), full spine on leaf and petals, low seed yield and low seed oil content. Therefore the present study aimed to assess the 
genetic evolution of promising mutant lines (earliness, spineless) for seed yield and oil content, and subsequently improvement of new commercial safflower varieties with economic beneficials in Egypt.

\section{MATERIALS AND METHODES}

\section{Materials}

This study continued during six generations (M5-M10 generations) for 27 mutant lines and their two parents (line III and line VI). These mutant lines were obtained from M.Sc. Thesis of Ahmed (2012) as a promising mutants, whereas spineless and earliness criteria (Table 1).

\section{Methods}

These lines were carried out at six generations from 2013 to 2018. The trial was laid out in randomized complete blocks design with 3 replications, accommodating 5 ridges, 60 $\mathrm{cm}$ apart, $4 \mathrm{~m}$ length with $15 \mathrm{~cm}$ plant to plant distance and seeding rate of $12 \mathrm{~kg}$ fad. Fertilization of nitrogen and phosphors were applied as common agriculture. Genotype seeds were sown by hand on $14^{\text {th }}$, November.

At M5 to M9, the spineless and days to maturity were recorded per each generation for stability study of these mutant lines. The criteria were recorded at M10 generation as follows: 1-plant height, 2-No. of branches per plant, 3-No.of capitula per plant, 4-No. of seeds per capitulam, 5-No. of seeds per plant, 6-100 seed weight (gm), 7-seed weight per plant (g), 8-Days to maturity, and 9- Oil content. Oil content of the samples was obtained using the soxhlet extraction method with hexane as described in AOAC (1990).

\section{Statistical Analysis}

The collected data were analysed using the randomized complete blocks design (RCBD) according to Gomez and Gomez (1984) as analysis of variance and heritability estimates were recorded in the basis of Singh and Chaudary (1977). The correlation coefficients were estimated between all criteria.

\section{RESULTIS AND DISCUSSION}

The stability and adaptability values of mutant lines under study were shown in Table 2.
The results showed that line VI was more stable than line III, because of the death of 3 mutant lines only from 9 mutant lines, by comparison, it was 10 out of 18 for line III throw recurrent six years. Therefore, line VI and their mutants are more survival and adaptable than line III and their mutants. For homozygosity of spineless criteria at 3 generations (M8, M9 and M10 generation) where shown in two mutant lines, line 4 and line 7 at line III genotype. At line VI, two mutant lines (line 2 and line 5) were stable and adaptable at 3 years (M8, M9 and M10 generation). In general, spine formation is considered as a polygenic character, but the spineless mutant lines at line VI and line III over recurrent three years may confirm the likelihood of homozygous genotypes for this criterion. The survival of studied genotypes for days to maturity was shown in Table 3 . In contrast, multiple mutant lines had the same days to maturity at 4 generations (4 years): Fortunately, the stable and adaptable mutant lines for spineless possessed earliness stability at 4 generations. These lines were line 4 , and line 7 at line III genotype and line 2 and line 5 at line VI genotype. Moreover, survival mutant lines in two line genotypes and their mutants possessed high stablility, for days to maturity. These results weighted the simple heritable system of days to maturity, than the spineless criteria. These promising mutant lines had 168 and 170 of line 4 and 7 from line III and 177 and 182 days of line 2 and 5 from line VI by comparison to 180 and 188 days for control of line III and line VI, respectively. These results suggest the possibility of new inhancement, in Egyptian varieties for cultivation of harsh and poor land desert. These varities possessed spineless and earliness and so they can be used as forage crop.

These results are agreeded with Ragab et al. (2008), they were studying spineless safflower mutant lines for seed oil content and fatty acid profiles. The results showed a changeable for these mutant lines than the mother variety in multiple criteria especially oil content.

Highly significant difference between genotypes of line III and their mutant lines for nine characters and line VI and their mutant lines for nine characters under study were shown in Tables 4 and 6 . High broad sense heritability $\left(h^{2}\right)$ of No. of capitula per plant, 100 -seed weight, 
Table 1. Pedigree of mutant lines and their parents at fourth generation used in the present study $(*)$

\begin{tabular}{|c|c|c|}
\hline No. of line & Pedigree & Source of mutant \\
\hline \multicolumn{3}{|l|}{ Line III } \\
\hline 1 & $(3)-A-5-5$ & $\gamma$-ray 100Gy ( spinyless - early) \\
\hline 2 & (3)-B-7-2 & $\gamma$-ray 100Gy ( tip spine - early) \\
\hline 3 & (4)-A-1-10 & $\gamma$-ray $100 \mathrm{~Gy}$ ( tip spine - early) \\
\hline 4 & (4)-B-2-8 & $\gamma$-ray $100 \mathrm{~Gy}$ ( tip spine - early) \\
\hline 5 & (4)-B-6-6 & $\gamma$-ray $100 \mathrm{~Gy}$ ( tip spine - normal) \\
\hline 6 & $(5)-A-2-5$ & $\gamma$-ray 100 Gy ( tip spine - normal) \\
\hline 7 & (5)-A-10-7 & $\gamma$-ray 100 Gy ( tip spine - normal) \\
\hline 8 & (5)-A-10-1 & $\gamma$-ray 150Gy ( spinyless - early) \\
\hline 9 & $(5)-B-3-4$ & $\gamma$-ray $150 \mathrm{~Gy}$ ( spinyless - early) \\
\hline 10 & (5)-B-5-2 & $\gamma$-ray $150 \mathrm{~Gy}$ ( spinyless - early) \\
\hline 11 & $(6)-A-7-6$ & $\gamma$-ray $150 \mathrm{~Gy}$ ( spinyless - early) \\
\hline 12 & $(7)-A-5-5$ & $\gamma$-ray $150 \mathrm{~Gy}$ ( spinyless - early) \\
\hline 13 & $(2)-A-3-7$ & $\gamma$-ray 150 Gy ( spinyless - early) \\
\hline 14 & (3)-A-7-3 & $\gamma$-ray 150 Gy ( spinyless - early) \\
\hline 15 & $(5)-A-1-2$ & $\gamma$-ray 200Gy ( spinyless - early) \\
\hline 16 & $(5)-B-1-4$ & $\gamma$-ray 200Gy ( spinyless - early) \\
\hline 17 & (6)-B-3-12 & $\gamma$-ray 200 Gy ( tip spine - normal) \\
\hline 18 & (7)-A-2-4 & $\gamma$-ray 200 Gy ( tip spine - normal) \\
\hline \multicolumn{3}{|l|}{ Line VI } \\
\hline 1 & $(2)-A-5-1$ & $\gamma$-ray 100Gy ( spinyless - normal ) \\
\hline 2 & (2)-A-7-8 & $\gamma$-ray 100Gy ( spinyless - normal ) \\
\hline 3 & (2)-B-2-10 & $\gamma$-ray 100Gy ( spinyless - normal ) \\
\hline 4 & (3)-A-1-2 & $\gamma$-ray 150Gy ( spinyless - normal ) \\
\hline 5 & $(3)-A-7-3$ & $\gamma$-ray 150Gy ( spinyless - normal ) \\
\hline 6 & $(7)-A-2-4$ & NaN3:0.003M 3hs ( spinyless - early ) \\
\hline 7 & $(7)-A-6-2$ & NaN3:0.003M 4hs ( spinyless - normal ) \\
\hline 8 & (6)-A-1-5 & NaN3:0.003M 4hs ( spinyless - normal ) \\
\hline 9 & $(7)-A-5-2$ & NaN3:0.003M 4hs ( spinyless - normal ) \\
\hline
\end{tabular}

Note: for example (3)-A-5-5, First number (3) as second generation,

Third No. (A-5) as third generation, (5) as No. of fourth generation.

*These materials from M.Sc. thesis of Marwa Ahmed ( 2012) 
Table 2. The stability of safflower line III, line VI and their promising mutants for spine formation at M5 to M10 generations

\begin{tabular}{|c|c|c|c|c|c|c|c|c|c|c|c|c|c|c|c|c|c|c|}
\hline \multirow{2}{*}{$\begin{array}{l}\text { No. of } \\
\text { line }\end{array}$} & \multirow[t]{2}{*}{ Genotype } & \multicolumn{3}{|c|}{ M5 } & \multicolumn{3}{|c|}{ M6 } & \multicolumn{3}{|c|}{ M7 } & \multicolumn{3}{|c|}{ M8 } & \multicolumn{3}{|c|}{ M9 } & \multicolumn{2}{|r|}{ M10 } \\
\hline & & $\mathbf{3}$ & $\mathbf{T}$ & $\mathbf{F}$ & $\mathbf{S}$ & $\mathbf{T}$ & $\mathbf{F}$ & $\mathbf{S}$ & $\mathbf{T}$ & $\mathbf{F}$ & $\mathbf{S}$ & $\mathbf{T}$ & $\mathbf{F}$ & $\mathbf{S}$ & $\mathbf{T}$ & $\mathbf{F}$ & $\mathbf{S}$ & $\begin{array}{ll}\mathbf{T} & \mathbf{F}\end{array}$ \\
\hline
\end{tabular}

Line III Control

$\begin{array}{llllllllllllllllllll}1 & (3)-\mathrm{A}-5-5 & \mathrm{~S} & \mathrm{~T} & - & \mathrm{S} & \mathrm{T} & \mathrm{F} & - & \mathrm{T} & \mathrm{F} & - & - & \mathrm{F} & \mathrm{D} & \mathrm{D} & \mathrm{D} & \mathrm{D} & \mathrm{D} & \mathrm{D} \\ \mathbf{2} & (3)-\mathrm{B}-7-2 & \mathrm{~S} & - & - & \mathrm{D} & \mathrm{D} & \mathrm{D} & \mathrm{D} & \mathrm{D} & \mathrm{D} & \mathrm{D} & \mathrm{D} & \mathrm{D} & \mathrm{D} & \mathrm{D} & \mathrm{D} & \mathrm{D} & \mathrm{D} & \mathrm{D} \\ \mathbf{3} & (4)-\mathrm{A}-1-10 & \mathrm{~S} & - & - & \mathrm{D} & \mathrm{D} & \mathrm{D} & \mathrm{D} & \mathrm{D} & \mathrm{D} & \mathrm{D} & \mathrm{D} & \mathrm{D} & \mathrm{D} & \mathrm{D} & \mathrm{D} & \mathrm{D} & \mathrm{D} & \mathrm{D} \\ \mathbf{4} & (4)-\mathrm{B}-2-8 & \mathrm{~S} & - & - & \mathrm{S} & \mathrm{T} & \mathrm{F} & \mathrm{S} & \mathrm{T} & - & \mathrm{S} & - & - & \mathrm{S} & - & - & \mathrm{S} & - & - \\ \mathbf{5} & (4)-\mathrm{B}-6-6 & \mathrm{~S} & - & - & \mathrm{D} & \mathrm{D} & \mathrm{D} & \mathrm{D} & \mathrm{D} & \mathrm{D} & \mathrm{D} & \mathrm{D} & \mathrm{D} & \mathrm{D} & \mathrm{D} & \mathrm{D} & \mathrm{D} & \mathrm{D} & \mathrm{D} \\ \mathbf{6} & (5)-\mathrm{A}-2-5 & \mathrm{~S} & - & - & \mathrm{D} & \mathrm{D} & \mathrm{D} & \mathrm{D} & \mathrm{D} & \mathrm{D} & \mathrm{D} & \mathrm{D} & \mathrm{D} & \mathrm{D} & \mathrm{D} & \mathrm{D} & \mathrm{D} & \mathrm{D} & \mathrm{D} \\ \mathbf{7} & (5)-\mathrm{A}-10-7 & \mathrm{~S} & - & - & \mathrm{S} & \mathrm{T} & \mathrm{F} & \mathrm{S} & \mathrm{T} & - & \mathrm{S} & - & - & \mathrm{S} & - & - & \mathrm{S} & - & - \\ \mathbf{8} & (5)-\mathrm{A}-10-1 & \mathrm{~S} & - & - & \mathrm{S} & \mathrm{T} & - & \mathrm{S} & \mathrm{T} & - & \mathrm{S} & - & - & \mathrm{D} & \mathrm{D} & \mathrm{D} & \mathrm{D} & \mathrm{D} & \mathrm{D} \\ \mathbf{9} & (5)-\mathrm{B}-3-4 & \mathrm{~S} & - & - & \mathrm{S} & \mathrm{T} & - & \mathrm{S} & \mathrm{T} & - & \mathrm{S} & - & - & - & \mathrm{T} & - & \mathrm{S} & - & - \\ \mathbf{1 0} & (5)-\mathrm{B}-5-2 & \mathrm{~S} & - & - & \mathrm{S} & \mathrm{T} & \mathrm{F} & \mathrm{S} & - & - & \mathrm{S} & \mathrm{T} & - & \mathrm{S} & - & - & \mathrm{S} & - & - \\ \mathbf{1 1} & (6)-\mathrm{A}-7-6 & \mathrm{~S} & - & - & \mathrm{S} & \mathrm{T} & \mathrm{F} & \mathrm{D} & \mathrm{D} & \mathrm{D} & \mathrm{D} & \mathrm{D} & \mathrm{D} & \mathrm{D} & \mathrm{D} & \mathrm{D} & \mathrm{D} & \mathrm{D} & \mathrm{D} \\ \mathbf{1 2} & (7)-\mathrm{A}-5-5 & \mathrm{~S} & - & - & \mathrm{S} & \mathrm{T} & \mathrm{F} & \mathrm{S} & \mathrm{T} & - & \mathrm{S} & - & - & \mathrm{S} & \mathrm{T} & - & \mathrm{S} & - & - \\ \mathbf{1 3} & (2)-\mathrm{A}-3-7 & \mathrm{D} & \mathrm{D} & \mathrm{D} & \mathrm{D} & \mathrm{D} & \mathrm{D} & \mathrm{D} & \mathrm{D} & \mathrm{D} & \mathrm{D} & \mathrm{D} & \mathrm{D} & \mathrm{D} & \mathrm{D} & \mathrm{D} & \mathrm{D} & \mathrm{D} & \mathrm{D} \\ \mathbf{1 4} & (3)-\mathrm{A}-7-3 & \mathrm{~S} & - & - & - & \mathrm{T} & \mathrm{F} & \mathrm{S} & - & \mathrm{F} & - & \mathrm{T} & - & \mathrm{S} & \mathrm{T} & - & \mathrm{S} & - & - \\ \mathbf{1 5} & (5)-\mathrm{A}-1-2 & - & \mathrm{T} & - & \mathrm{S} & \mathrm{T} & - & \mathrm{D} & \mathrm{D} & \mathrm{D} & \mathrm{D} & \mathrm{D} & \mathrm{D} & \mathrm{D} & \mathrm{D} & \mathrm{D} & \mathrm{D} & \mathrm{D} & \mathrm{D} \\ \mathbf{1 6} & (5)-\mathrm{B}-1-4 & \mathrm{~S} & - & - & \mathrm{D} & \mathrm{D} & \mathrm{D} & \mathrm{D} & \mathrm{D} & \mathrm{D} & \mathrm{D} & \mathrm{D} & \mathrm{D} & \mathrm{D} & \mathrm{D} & \mathrm{D} & \mathrm{D} & \mathrm{D} & \mathrm{D} \\ \mathbf{1 7} & (6)-\mathrm{B}-3-12 & \mathrm{~S} & - & - & \mathrm{S} & \mathrm{T} & \mathrm{F} & \mathrm{S} & - & - & - & \mathrm{T} & - & \mathrm{S} & \mathrm{T} & - & \mathrm{S} & - & - \\ \mathbf{1 8} & (7)-\mathrm{A}-2-4 & \mathrm{~S} & - & - & \mathrm{S} & \mathrm{T} & \mathrm{F} & \mathrm{S} & \mathrm{T} & \mathrm{F} & \mathrm{S} & - & - & \mathrm{S} & \mathrm{T} & - & \mathrm{S} & - & -\end{array}$

Line VI Control - - F - - F - - F - - F - $-F-$ F

\begin{tabular}{llllllllllllllllllll}
1 & $(2)-\mathrm{A}-5-1$ & $\mathrm{~S}$ & - & - & $\mathrm{S}$ & $\mathrm{T}$ & $\mathrm{F}$ & $\mathrm{S}$ & - & - & $\mathrm{S}$ & $\mathrm{T}$ & - & $\mathrm{S}$ & - & - & $\mathrm{S}$ & - & - \\
2 & $(2)-\mathrm{A}-7-8$ & $\mathrm{~S}$ & - & - & $\mathrm{S}$ & $\mathrm{T}$ & $\mathrm{F}$ & $\mathrm{S}$ & - & - & $\mathrm{S}$ & - & - & $\mathrm{S}$ & - & - & $\mathrm{S}$ & - & - \\
3 & $(2)-\mathrm{B}-2-10$ & $\mathrm{~S}$ & - & - & $\mathrm{S}$ & $\mathrm{T}$ & $\mathrm{F}$ & $\mathrm{D}$ & $\mathrm{D}$ & $\mathrm{D}$ & $\mathrm{D}$ & $\mathrm{D}$ & $\mathrm{D}$ & $\mathrm{D}$ & $\mathrm{D}$ & $\mathrm{D}$ & $\mathrm{D}$ & $\mathrm{D}$ & $\mathrm{D}$ \\
4 & $(3)-\mathrm{A}-1-2$ & $\mathrm{~S}$ & - & - & $\mathrm{S}$ & $\mathrm{T}$ & - & $\mathrm{S}$ & - & - & - & $\mathrm{T}$ & - & $\mathrm{D}$ & $\mathrm{D}$ & $\mathrm{D}$ & $\mathrm{D}$ & $\mathrm{D}$ & $\mathrm{D}$ \\
5 & $(3)-\mathrm{A}-7-3$ & $\mathrm{~S}$ & - & - & $\mathrm{S}$ & $\mathrm{T}$ & - & $\mathrm{S}$ & $\mathrm{T}$ & - & $\mathrm{S}$ & - & - & $\mathrm{S}$ & - & - & $\mathrm{S}$ & - & - \\
6 & $(7)-\mathrm{A}-2-4$ & $\mathrm{~S}$ & - & - & $\mathrm{S}$ & $\mathrm{T}$ & - & $\mathrm{S}$ & - & - & $\mathrm{S}$ & - & $\mathrm{F}$ & $\mathrm{S}$ & - & - & $\mathrm{S}$ & - & - \\
7 & $(7)-\mathrm{A}-6-2$ & $\mathrm{~S}$ & - & - & $\mathrm{S}$ & $\mathrm{T}$ & $\mathrm{F}$ & $\mathrm{S}$ & - & - & $\mathrm{S}$ & $\mathrm{T}$ & - & $\mathrm{S}$ & - & - & $\mathrm{S}$ & - & - \\
8 & $(6)-\mathrm{A}-1-5$ & $\mathrm{~S}$ & - & - & - & $\mathrm{T}$ & $\mathrm{F}$ & $\mathrm{D}$ & $\mathrm{D}$ & $\mathrm{D}$ & $\mathrm{D}$ & $\mathrm{D}$ & $\mathrm{D}$ & $\mathrm{D}$ & $\mathrm{D}$ & $\mathrm{D}$ & $\mathrm{D}$ & $\mathrm{D}$ & $\mathrm{D}$ \\
9 & (7)-A-5-2 & $\mathrm{S}$ & - & - & $\mathrm{S}$ & $\mathrm{T}$ & $\mathrm{F}$ & $\mathrm{S}$ & $\mathrm{T}$ & - & $\mathrm{S}$ & $\mathrm{T}$ & - & $\mathrm{S}$ & $\mathrm{T}$ & - & $\mathrm{S}$ & - & - \\
\hline
\end{tabular}


Table 3. The stability of safflower line III, line VI and their promising mutants for days to maturity at M5 to M10 generations

\begin{tabular}{|c|c|c|c|c|c|c|c|}
\hline $\begin{array}{l}\text { No. of } \\
\text { line }\end{array}$ & Genotype & M5 & M6 & M7 & M8 & M9 & M10 \\
\hline Line III & Control & 180 & 180 & 180 & 180 & 180 & 180 \\
\hline 1 & (3)-A-5-5 & 175 & 170 & 168 & $\mathrm{D}$ & $\mathrm{D}$ & $\mathrm{D}$ \\
\hline 2 & (3)-B-7-2 & 180 & $\mathrm{D}$ & $\mathrm{D}$ & $\mathrm{D}$ & $\mathrm{D}$ & $\mathrm{D}$ \\
\hline 3 & (4)-A-1-10 & 180 & $\mathrm{D}$ & $\mathrm{D}$ & $\mathrm{D}$ & $\mathrm{D}$ & $\mathrm{D}$ \\
\hline 4 & (4)-B-2-8 & 180 & 175 & 168 & 168 & 168 & 168 \\
\hline 5 & (4)-B-6-6 & 180 & $\mathrm{D}$ & $\mathrm{D}$ & $\mathrm{D}$ & $\mathrm{D}$ & $\mathrm{D}$ \\
\hline 6 & (5)-A-2-5 & 180 & $\mathrm{D}$ & D & $\mathrm{D}$ & D & D \\
\hline 7 & (5)-A-10-7 & 180 & 180 & 170 & 170 & 170 & 170 \\
\hline 8 & (5)-A-10-1 & 170 & 180 & 166 & 166 & D & D \\
\hline 9 & (5)-B-3-4 & 170 & 180 & 168 & 168 & 168 & 168 \\
\hline 10 & (5)-B-5-2 & 163 & 165 & 168 & 168 & 168 & 168 \\
\hline 11 & (6)-A-7-6 & 175 & 175 & $\mathrm{D}$ & $\mathrm{D}$ & $\mathrm{D}$ & $\mathrm{D}$ \\
\hline 12 & (7)-A-5-5 & 175 & 175 & 172 & 172 & 172 & 172 \\
\hline 13 & (2)-A-3-7 & $\mathrm{D}$ & D & D & $\mathrm{D}$ & D & $\mathrm{D}$ \\
\hline 14 & (3)-A-7-3 & 170 & 170 & 172 & 172 & 172 & 172 \\
\hline 15 & (5)-A-1-2 & 175 & 180 & D & D & D & D \\
\hline 16 & (5)-B-1-4 & 180 & $\mathrm{D}$ & D & $\mathrm{D}$ & $\mathrm{D}$ & $\mathrm{D}$ \\
\hline 17 & (6)-B-3-12 & 175 & 170 & 175 & 175 & 175 & 175 \\
\hline 18 & (7)-A-2-4 & 175 & 170 & 175 & 175 & 175 & 175 \\
\hline Line VI & Control & 188 & 188 & 188 & 188 & 188 & 188 \\
\hline 1 & (2)-A-5-1 & 177 & 177 & 177 & 182 & 177 & 177 \\
\hline 2 & (2)-A-7-8 & 182 & 182 & 177 & 177 & 177 & 177 \\
\hline 3 & (2)-B-2-10 & 177 & 177 & D & D & $\mathrm{D}$ & D \\
\hline 4 & (3)-A-1-2 & 177 & 177 & 177 & 177 & $\mathrm{D}$ & $\mathrm{D}$ \\
\hline 5 & (3)-A-7-3 & 177 & 177 & 182 & 182 & 182 & 182 \\
\hline 6 & (7)-A-2-4 & 177 & 177 & 180 & 177 & 182 & 182 \\
\hline 7 & (7)-A-6-2 & 177 & 177 & 177 & 177 & 177 & 177 \\
\hline 8 & (6)-A-1-5 & 177 & 177 & $\mathrm{D}$ & D & $\mathrm{D}$ & $\mathrm{D}$ \\
\hline 9 & (7)-A-5-2 & 177 & 177 & 180 & 182 & 180 & 180 \\
\hline
\end{tabular}


Table 4. Mean sum of squares (MS) and heritability $\left(h^{2}\right)$ of morphological, quantitive and oil content of safflower for line III and their promising mutant lines at M10 generations

\begin{tabular}{|c|c|c|c|c|c|c|c|c|c|c|}
\hline \multirow[t]{2}{*}{$\begin{array}{l}\text { Source of } \\
\text { variation }\end{array}$} & \multirow[t]{2}{*}{$\overline{\text { d.f }}$} & $\begin{array}{c}\text { Plant } \\
\text { height } \\
\text { (cm) }\end{array}$ & $\begin{array}{c}\text { No. of } \\
\text { branches } \\
\text { per plant }\end{array}$ & $\begin{array}{c}\text { No. of } \\
\text { capitula } \\
\text { per plant }\end{array}$ & $\begin{array}{c}\text { No. of } \\
\text { seeds per } \\
\text { capitulam }\end{array}$ & $\begin{array}{c}\text { No. of } \\
\text { seeds per } \\
\text { plant }\end{array}$ & $\begin{array}{c}100 \text { seed } \\
\text { weight per } \\
\text { plant (g) }\end{array}$ & $\begin{array}{l}\text { Seed weight } \\
\text { per plant } \\
\text { (g) }\end{array}$ & $\begin{array}{c}\text { Days } \\
\text { to } \\
\text { maturity }\end{array}$ & $\begin{array}{l}\text { Oil } \\
\text { (\%) }\end{array}$ \\
\hline & & MS & MS & MS & MS & MS & MS & MS & MS & MS \\
\hline$\overline{\text { Replication }}$ & 3 & $883.33^{* *}$ & $45.11 * *$ & $929.58^{* *}$ & $661.07^{* *}$ & $3020868.86^{* *}$ & $8.9^{* *}$ & $5554.22^{* *}$ & $26.89 * *$ & $16.25^{* *}$ \\
\hline Treatment & 8 & $566.94 * *$ & $19.72 * *$ & $763.4^{* *}$ & $180.44^{* *}$ & $1319106.37 * *$ & $4.09 * *$ & $1246.28^{* *}$ & $62.00 * *$ & $35.28 * *$ \\
\hline Error & 24 & 89.6 & 2.79 & 47.13 & 32.85 & 204517.26 & 0.27 & 140.8 & 3.81 & 3.5 \\
\hline$h^{2}$ in broad sc & nse & $57.11 \%$ & $58.50 \%$ & $79.16 \%$ & $52.89 \%$ & $57.67 \%$ & $77.95 \%$ & $94.91 \%$ & $79.23 \%$ & $69.41 \%$ \\
\hline
\end{tabular}

*Significant at $0.05 .,{ }^{* *}$ Significant at 0.01 .

seed weight per plant, days to maturity and oil content were $79.16 \%, 77.95 \%, 94.91 \%, 79.23 \%$ and $69.41 \%$, respectively among line III and their mutant lines. Among line VI and their mutants, high heritability were found for almost criteria expect No. of branches per plant. The fluctuation of heritability, estimates among line III and line VI and their mutants was detected because the variation among line VI and their mutants was larger than the line III and their mutants. So, these results may be important for line VI and their mutants, which, they are recorded as a stable and adaptable. These results showed that the high estimation of heritability increases the efficiency of selection for a special trait. The selection of high oil content and good seed yield could be effective for development of new genotypes possessing high oil content and seed yield. Heritability is a good indicator of the transmission of traits from parents to progeny. The assessment of heritability helps in selection of the best genotypes from a varied genetic population, Reddy et al. (2013). They also reported that heritability, could be grouped as low (below 30\%), medium (30-60\%) and high (above 60\%). Tahernezhad et al. (2018), studied the broad sense heritability of safflower genotypes for many criteria. They classified it into groups on the basis of their heritability; group1 had high heritability and comprised plant height, days to flowering, 1000-seed weight, number of seed per capitula. These traits are less influenced by the environment and are strongly controlled by genetic factors. The highest broad sense heritability was estimated for plant height, which is in accordance with the results of Mozaffri and Asadi (2006), Camas and Esendal (2006) and Elfadl et al (2010). The present results confirmed with the above results in many criteria under studies.

Average mean of quantitative characters and oil content were shown in Table 5 of line III and their mutants. These results showed that line 4 and 7 are considered as earliness (168 and 170 days to maturity) with no effect in seed yield and its components, but, these lines possessed low oil content. Very important line was reported of line 14 for significant oil content and subsequently, it facted high oil content, with no effect of seed yield and its components. In contrast lines 12 and 17 had high oil content, with low seed yield and its components.

So, line VI and their mutants exhibited a large variation and excellent genotypes may be selected for genetic improvement of oil content and seed yield (Table 7). Line 2 had 23.75 with comparison to 20.10 oil content, as well as earliness (177) and highly significant of No. of seeds per capitulum. In addition, line 6 had highly significance for 100 -seed weight. Interesting remark, line 7 possessed short stature (138.25 $\mathrm{cm})$ with comparison of mother plant $(210 \mathrm{~cm})$. Reduced plant height is an important trait in plant breeding, mainly because short genotype is more resistant to lodging than standard types (Austin et al., 1980; Fick and Miller, 1997).

Relationship among studied traits using correlation coefficient was recorded in Tables 8,9 and 10 of line III and line VI and their mutants and all most genotypes of line III and line VI and their mutants, respectively. Maluszynski et al. (2002) stated that induced mutation has been extensively used for creating new genetic variation in crop plant. 
Table 5. Average mean of morphological, quantitive and oil content of safflower for line III and their promising mutant line at M10 generation

\begin{tabular}{|c|c|c|c|c|c|c|c|c|c|c|}
\hline No. of line & $\mathbf{G}$ & $\begin{array}{c}\text { Plant } \\
\text { height } \\
(\mathrm{cm})\end{array}$ & $\begin{array}{c}\text { No. of } \\
\text { branches } \\
\text { per plant }\end{array}$ & $\begin{array}{c}\text { No. of } \\
\text { capitula } \\
\text { per plant }\end{array}$ & $\begin{array}{c}\text { No. of } \\
\text { seeds per } \\
\text { capitulam }\end{array}$ & $\begin{array}{c}\text { No. of } \\
\text { seeds per } \\
\text { plant }\end{array}$ & $\begin{array}{l}100 \text { seed } \\
\text { weight per } \\
\text { plant }(\mathrm{g})\end{array}$ & $\begin{array}{l}\text { Seed weight } \\
\text { per plant } \\
\text { (g) }\end{array}$ & $\begin{array}{c}\text { Days } \\
\text { to } \\
\text { maturity }\end{array}$ & $\begin{array}{c}\text { Oil } \\
(\%)\end{array}$ \\
\hline Control III & Control & 199.00 & 12.11 & 48.00 & 29.25 & 1600.00 & 7.86 & 78.25 & 180 & 18.00 \\
\hline 4 & (4).B.2.8 & 183.25 & 9.25 & 49.00 & 37.00 & 1825.00 & 4.96 & 66.75 & 168 & 14.00 \\
\hline 7 & (5).A.10.7 & 210.00 & 11.00 & 44.25 & 39.75 & 1885.25 & 5.70 & 76.75 & 170 & 18.00 \\
\hline 9 & (5).B.3.4 & 198.00 & 15.00 & 55.75 & 39.50 & 2499.55 & 5.50 & 80.00 & 168 & 16.00 \\
\hline 10 & (5).B.5.2 & 193.75 & 12.25 & 48.00 & 34.25 & 1621.00 & 7.50 & 83.25 & 168 & 20.00 \\
\hline 12 & (7).A.5.5 & 185.00 & 11.75 & 28.75 & 19.75 & 593.25 & 5.63 & 31.75 & 172 & 22.00 \\
\hline 14 & (3).A.7.3 & 203.25 & 10 & 25.25 & 41.00 & 1137.25 & 6.56 & 81.75 & 172 & 23.00 \\
\hline 17 & (6).B.3.12 & 221.75 & 9.25 & 41.00 & 34.00 & 1442.00 & 5.48 & 57.75 & 175 & 18.00 \\
\hline 18 & (7).A. 2.4 & 196.75 & 12.25 & 31.00 & 32.33 & 1008.25 & 6.9 & 57.75 & 172 & 21.00 \\
\hline Average & & 198.97 & 11.42 & 41.22 & 34.09 & 1512.39 & 6.23 & 68.22 & 171.66 & 18.88 \\
\hline LSD & & 13.80 & 2.47 & 10.01 & 8.35 & 661.60 & 0.74 & 17.31 & 2.84 & 2.72 \\
\hline
\end{tabular}

Table 6. Mean sum of squares (MS) and heritability $\left(h^{2}\right)$ of morphological, quantitive and oil content of safflower for line VI and their promising mutant lines at M10 generation

\begin{tabular}{|c|c|c|c|c|c|c|c|c|c|c|}
\hline \multirow[t]{2}{*}{$\begin{array}{l}\text { Source of } \\
\text { variation }\end{array}$} & \multirow[t]{2}{*}{ d.f } & \multirow{2}{*}{$\begin{array}{c}\begin{array}{c}\text { Plant } \\
\text { height } \\
(\mathrm{cm})\end{array} \\
\text { MS }\end{array}$} & \multirow{2}{*}{$\begin{array}{c}\begin{array}{c}\text { No. of } \\
\text { branches } \\
\text { per plant }\end{array} \\
\text { MS }\end{array}$} & $\begin{array}{c}\text { No. of } \\
\text { capitula } \\
\text { per plant }\end{array}$ & $\begin{array}{c}\text { No. of } \\
\text { seeds per } \\
\text { capitulam }\end{array}$ & \multirow{2}{*}{$\begin{array}{c}\begin{array}{c}\text { No. of } \\
\text { seeds per } \\
\text { plant }\end{array} \\
\text { MS }\end{array}$} & \multirow{2}{*}{$\begin{array}{c}\begin{array}{c}100 \text { seed } \\
\text { weight per } \\
\text { plant }(\mathrm{g})\end{array} \\
\text { MS }\end{array}$} & \multirow{2}{*}{$\begin{array}{c}\text { Seed weight } \\
\text { per plant } \\
\text { (g) }\end{array}$} & \multirow{2}{*}{$\begin{array}{c}\begin{array}{c}\text { Days } \\
\text { to } \\
\text { maturity }\end{array} \\
\text { MS }\end{array}$} & \multirow{2}{*}{\begin{tabular}{|c|} 
Oil \\
$(\%)$ \\
MS
\end{tabular}} \\
\hline & & & & MS & MS & & & & & \\
\hline ion & 3 & $493.33 * *$ & $59.43^{* *}$ & $551.29 * *$ & $203.85^{* *}$ & $1068359.81 * *$ & $2.87 * *$ & $1646.14 * *$ & $28.81 * *$ & $9.23^{\circ}$ \\
\hline Trea & 6 & $3699.5 * *$ & $72.12 * *$ & $581.31 * *$ & $174.39 * *$ & 1093613 & $2.72 * *$ & $1580.66^{* *}$ & $60.57 * *$ & $7.28 *$ \\
\hline Error & 18 & 55.39 & 11.15 & 54.23 & 7.345 & 128975.48 & 0.19 & 137.12 & 1.14 & 3.62 \\
\hline \multicolumn{2}{|c|}{$h^{2}$ in broad sense } & $94.26 \%$ & $57.74 \%$ & $70.84 \%$ & $85.04 \%$ & $65.15 \%$ & $76.82 \%$ & $72.46 \%$ & $92.89 \%$ & $61.99^{\circ}$ \\
\hline
\end{tabular}

Table 7. Average mean of morphological, quantitive and oil content of safflower for line VI and promising mutant line at M10 generation

\begin{tabular}{|c|c|c|c|c|c|c|c|c|c|c|}
\hline $\begin{array}{l}\text { No. of } \\
\text { line }\end{array}$ & Genotype & $\begin{array}{c}\text { Plant } \\
\text { height } \\
(\mathrm{cm})\end{array}$ & $\begin{array}{c}\text { No. of } \\
\text { branches } \\
\text { per plant }\end{array}$ & $\begin{array}{c}\text { No. of } \\
\text { capitula } \\
\text { per plant }\end{array}$ & $\begin{array}{c}\text { No. of } \\
\text { seeds per } \\
\text { capitulam }\end{array}$ & $\begin{array}{c}\text { No. of } \\
\text { seeds per } \\
\text { plant }\end{array}$ & $\begin{array}{c}100 \text { seed } \\
\text { weight per } \\
\text { plant }(\mathrm{g})\end{array}$ & $\begin{array}{c}\text { Seed weight } \\
\text { per plant } \\
\text { (g) }\end{array}$ & $\begin{array}{c}\text { Days } \\
\text { to } \\
\text { maturity }\end{array}$ & $\begin{array}{c}\text { Oil } \\
(\%)\end{array}$ \\
\hline $\begin{array}{l}\text { Control } \\
\text { VI }\end{array}$ & Control & 210.00 & 9.80 & 39.96 & 39.10 & 1500.00 & 5.43 & 68.00 & 188 & 20.10 \\
\hline 1 & (2).A.5.1 & 203.25 & 14.00 & 40.50 & 42.00 & 1754.50 & 5.86 & 81.75 & 177 & 19.00 \\
\hline 2 & (2).A.7.8 & 200.00 & 6.25 & 23.00 & 47.25 & 1087.25 & 5.33 & 51.75 & 177 & 23.75 \\
\hline 5 & (3).A.7.3 & 236.25 & 9.25 & 43.00 & 35.25 & 1520.50 & 4.76 & 61.75 & 182 & 18.25 \\
\hline 6 & (7).A.2.4 & 199.00 & 7.00 & 32.25 & 34.75 & 1113.25 & 7.26 & 46.75 & 182 & 22.00 \\
\hline 9 & (7).A.5.2 & 203.25 & 17.25 & 42.75 & 34.00 & 1579.25 & 5.66 & 68.33 & 180 & 20.25 \\
\hline 7 & (7).A.6.2 & 138.25 & 7.25 & 25.75 & 30.75 & 772.50 & 6.50 & 28.25 & 177 & 19.25 \\
\hline Average & & 198.57 & 10.11 & 35.31 & 37.58 & 1332.46 & 5.83 & 58.08 & 179.58 & 20.37 \\
\hline LSD & & 11.05 & 4.96 & 10.93 & 3.99 & 533.5 & 1.40 & 17.39 & 1.57 & 2.72 \\
\hline
\end{tabular}


Table 8. Correlation coefficients ( $r$ ) between morphological, quantitive characters and oil content for line III and their promising mutant lines at M10 generation

\begin{tabular}{|c|c|c|c|c|c|c|c|c|c|}
\hline & $\begin{array}{c}\text { Plant } \\
\text { height } \\
(\mathrm{cm})\end{array}$ & $\begin{array}{c}\text { No. of } \\
\text { branches } \\
\text { per plant }\end{array}$ & $\begin{array}{c}\text { No. of } \\
\text { capitula per } \\
\text { plant }\end{array}$ & $\begin{array}{c}\text { No. of } \\
\text { seeds per } \\
\text { capitulam }\end{array}$ & $\begin{array}{c}\text { No. of } \\
\text { seeds per } \\
\text { plant }\end{array}$ & $\begin{array}{c}100 \text { seed } \\
\text { weight per } \\
\text { plant }(\mathrm{g})\end{array}$ & $\begin{array}{l}\text { Seed weight } \\
\text { per plant } \\
\text { (g) }\end{array}$ & $\begin{array}{c}\text { Days } \\
\text { to } \\
\text { maturity }\end{array}$ & $\begin{array}{r}\text { Oil } \\
(\%)\end{array}$ \\
\hline $\begin{array}{l}\text { Plant height (cm) } \\
\text { (c) }\end{array}$ & 1 & & & & & & & & \\
\hline No. of branches per plant & -0.18999 & 1 & & & & & & & \\
\hline No. of capitula per plant & -0.01069 & 0.59634 & 1 & & & & & & \\
\hline No. of seeds per capitulam & 0.384579 & -0.20051 & 0.102756 & 1 & & & & & \\
\hline No. of seeds per plant & 0.154191 & 0.419155 & $0.820804^{*}$ & 0.585235 & 1 & & & & \\
\hline 100 seed weight per plant (g) & -0.00552 & 0.510804 & 0.233239 & -0.16004 & -0.07214 & 1 & & & \\
\hline Seed weight per plant (g) & 0.23652 & 0.252031 & 0.486682 & $0.78233^{*}$ & $0.706959^{*}$ & 0.37683 & 1 & & \\
\hline Days to maturity & 0.357741 & 0.244312 & 0.229633 & -0.40445 & -0.16802 & 0.464061 & -0.13108 & 1 & \\
\hline Oil (\%) & 0.048002 & -0.05787 & $-0.70415^{*}$ & -0.32854 & $-0.78095^{*}$ & 0.441373 & -0.24951 & 0.204965 & 1 \\
\hline
\end{tabular}

Table 9. Correlation coefficients (r) between morphological, quantitive characters and oil content for line VI and their promising mutant lines at M10 generation

\begin{tabular}{|c|c|c|c|c|c|c|c|c|c|}
\hline & $\begin{array}{c}\text { Plant } \\
\text { height } \\
(\mathrm{cm})\end{array}$ & $\begin{array}{c}\text { No. of } \\
\text { branches } \\
\text { per plant }\end{array}$ & $\begin{array}{c}\text { No. of } \\
\text { capitula } \\
\text { per plant }\end{array}$ & $\begin{array}{c}\text { No. of } \\
\text { seeds per } \\
\text { capitulam }\end{array}$ & $\begin{array}{c}\text { No. of } \\
\text { seeds per } \\
\text { plant }\end{array}$ & $\begin{array}{c}100 \text { seed } \\
\text { weight per } \\
\text { plant (g) }\end{array}$ & $\begin{array}{l}\text { Seed weight } \\
\text { per plant } \\
\text { (g) }\end{array}$ & $\begin{array}{c}\text { Days } \\
\text { to } \\
\text { maturity }\end{array}$ & $\begin{array}{l}\text { Oil } \\
(\%)\end{array}$ \\
\hline Plant height (cm) & 1 & & & & & & & & \\
\hline No. of branches per plant & 0.157703 & 1 & & & & & & & \\
\hline No. of capitula per plant & 0.623717 & 0.283211 & 1 & & & & & & \\
\hline No. of seeds per capitulam & 0.436782 & -0.24272 & 0.258644 & 1 & & & & & \\
\hline No. of seeds per plant & 0.673646 & 0.272145 & $0.945751^{*}$ & 0.534343 & 1 & & & & \\
\hline 100 seed weight per plant (g) & -0.59854 & -0.16046 & -0.38758 & -0.42496 & -0.46588 & 1 & & & \\
\hline Seed weight per plant (g) & 0.720736 & 0.459583 & $0.818887^{*}$ & 0.576391 & $0.938377^{*}$ & -0.49132 & 1 & & \\
\hline Days to maturity & 0.319338 & -0.35844 & $0.601946^{*}$ & $0.73089^{*}$ & $0.71198^{*}$ & -0.07748 & 0.554386 & 1 & \\
\hline Oil (\%) & 0.037425 & -0.45701 & -0.23104 & 0.588352 & -0.05752 & 0.163197 & -0.06782 & 0.464857 & 1 \\
\hline
\end{tabular}

Table 10. Correlation coefficients (r) as general between morphological, quantitive characters and oil content for line III and line VI and their promising mutant lines at M10 generation

\begin{tabular}{|c|c|c|c|c|c|c|c|c|c|}
\hline & $\begin{array}{c}\text { Plant } \\
\text { height } \\
(\mathrm{cm})\end{array}$ & $\begin{array}{c}\text { No. of } \\
\text { branches } \\
\text { per plant }\end{array}$ & $\begin{array}{c}\text { No. of } \\
\text { capitula } \\
\text { per plant }\end{array}$ & $\begin{array}{c}\text { No. of } \\
\text { seeds per } \\
\text { capitulam }\end{array}$ & $\begin{array}{c}\text { No. of } \\
\text { seeds per } \\
\text { plant }\end{array}$ & $\begin{array}{c}100 \text { seed } \\
\text { weight per } \\
\text { plant }(\mathrm{g})\end{array}$ & $\begin{array}{l}\text { Seed weight } \\
\text { per plant } \\
\text { (g) }\end{array}$ & $\begin{array}{c}\text { Days } \\
\text { to } \\
\text { maturity }\end{array}$ & $\begin{array}{r}\text { Oil } \\
(\%)\end{array}$ \\
\hline Plant height (cm) & 1 & & & & & & & & \\
\hline No. of branches per plant & 0.070251 & 1 & & & & & & & \\
\hline No. of capitula per plant & 0.325329 & 0.432496 & 1 & & & & & & \\
\hline No. of seeds per capitulam & 0.365286 & -0.3009 & 0.07448 & 1 & & & & & \\
\hline No. of seeds per plant & 0.42571 & 0.325804 & $0.862658^{\text {** }}$ & 0.49557 & 1 & & & & \\
\hline 100 seed weight per plant (g) & -0.31398 & 0.197236 & 0.065226 & -0.3142 & -0.18609 & 1 & & & \\
\hline Seed weight per plant (g) & $0.518248^{*}$ & *0.415106 & $0.642771^{*}$ & $0.548157^{*}$ & $0.800377^{* *}$ & 0.061828 & 1 & & \\
\hline Days to maturity & 0.22017 & -0.30292 & 0.082433 & 0.305862 & 0.056559 & 0.003403 & -0.0428 & 1 & \\
\hline Oil (\%) & 0.040749 & -0.30809 & $-0.58344^{*}$ & 0.100273 & -0.54297 & 0.253417 & -0.23587 & 0.432307 & 1 \\
\hline
\end{tabular}


More than 2200 mutant varieties of different crops with improved agronomic traits have been developed and released to the farmers for general cultivation in the world. The present study confirmed that the relationship between characters under study varied between genotypes for example, oil content has slightly negative correlation with seed weight per plant and is different between genotypes (- 0.2495 ,0.0678 and -0.2358 ) for line III and line VI and for all, respectively. Moreover, the selection of oil content and seed weight per plant could be achieved in line VI and their mutants (- 0.0678). In addition, highly positive correlation between seed weight per plant and no.of capitula per plant and No. of seeds per plant at line VI (Table 9), but is different at line III (Table 8).

As shown in Table 10 positive and highly significant correlation between seed weight per plant and three component traits, i.e. No. of capitula, No. of seeds per capitulum and No. of seeds per plant. Therefore, these three component traits are considered as important for selection of high seed weight per plant. Many relationship change from genotypes to the other and this fact confirm the importance of mutagenic treatments for enhancement of genetic variation. These results go agree with others (Veena and Ravikumar, 2003; Pahlavani et al., 2005; Mozaffari and Asadi, 2006).

\section{REFERENCES}

Ahmed, Marwa E. (2012). Mutagenic and Biotechnological Studies on safflower (Carthamus tinctorius L.) M.Sc. Thesis. Genet. Dept., Fac. Agric., Zagazig Univ., Egypt.

AOAC (1990). Official Methods of Association of Official Analytical Chemists, $15^{\text {th }} \mathrm{Ed}$. AOAC Inc., Suite 4000, Virginia, USA.

Austin, R.B., J. Bingham, R.D. Blackwell, L.T. Evans, M.A. Ford, C.L. Morgan and M. Taylor (1980). Genetic improvement in winter wheat yields since 1900 and associated physiological chances. J. Agric. Sci., 94: $675-689$.

Camas, N. and E. Esendal (2006). Estimates of broad sense heritability for seed yield and yield components of safflower (Carthamus tinctorius L.). Hereditas, 143 : 55-57.
Chaturvedi, M., N. Srivastava, T. Gudipati, R. Bhadauria, S. Khandelwal and R.R. Das (2001). In vitro studies in the wild species of Carthamus-C. oxycantha L. In: Bergman J., Mündel HH (eds) Safflower: a multipurpose species with unexploited potential and world adaptability. Proc. $5^{\text {th }}$ Int. Safflower Conf., Williston, ND, Sidney, MT, 23-27 July, 47-49.

Claassen, C.E. (1950). Natural and controlled crossing in safflower (Carthamus tinctorius L.). Agron J 42:381-384.

Elfadl, E., C. Reinbrecht and W. Claupein (2010). Evaluation of phenotype variation in a world germplasm collection of safflower (Carthamus tinctorius L.) grown under organic farming conditions in Germany. Genet. Resour. Crop Evol., 57 (2): 155-170.

FAOS (2009). FAO Statistical Databases, http:// apps.fao.org.

FAOS (2014). FAO Statistical Databases, http:// apps.fao.org.

Fick, G.N. and J.F. Miller (1997). Sunflower breeding. In: A.A. Schneiter (ed.), Sunflower Technol. and Prod. Agron. Monograph, 35: 395-439.

Gomez, K.A. and A.A. Gomez (1984). Statistical procedures for Agricultural Research. $2^{\text {nd }} E d$. John Wiley and Sons. Inc. New York, USA, 680.

Khadeer, M.A. and S.Y. Anwar (1991). Induction and characterization of seed mutants for oil and fatty acid composition in safflower. Cytologia, 56 (4): 517-522.

Knowles, P.F. (1976) Safflower: Carthamus tinctorius (Compositae). J. Article, 31-33

Maluszynski, M.K.N., L. Van zanten and B.S. Ahloowalia (2002). Officially released mutant varieties. The FAO/Int. Atomic Energy Agency Database. Mut. Breed. Rev., 12 : 184.IAEA, Vienna, Austria.

Mozaffari, K. and A.A. Asadi (2006). Relationships among traits using correlation, principal components and path analysis in safflower mutants sown in irrigated and drought stress conditions. Asian J. Plant Sci., 5 (6): 977-983. 
Pahlavani, M.H., G. Saeidi and A.F. Mirlohi (2005). Genetic association among quantitative traits in safflower (Carthamus tinctorius, L.). Proc. $4^{\text {th }}$ Int. Iran, Russia Conf., $285: 288$

Ragab, A.I., M. Kassem and H.A.M. Moustafa (2008). Assesment of spineless safflower (Carthamus tinctorius, L.) mutant lines for seed oil content and fatty acid profiles. Plant Res. Dept. Nucl. Res. Centre. Atomic Energy Authority. Inchas, Egypt. INIS-EG-195

Ramaswamy, N.M. (2001). Safflower biotechnology: progress and prospects. In: Bergman J, Mündel HH (eds) Safflower: a multipurpose species with unexploited potential and world adaptability. Proc. $5^{\text {th }}$ Int. Safflower conference, Williston, ND, Sidney MT, 23-27 July, 59.

Reddy, M.P., B.N. Reddy, B.T. Arsul and J.J. Maheshwari (2013). Genetic variability, heritability and genetic advance of growth and yield components of linseed (Linum usitatissimum L.). Int. J. Curr. Microbiol App. Sci., 2 (9): 231-237.

Siddiqi, E.H., M. Ashraf and N.A. Akram (2007). Variation in seed germination and seedling growth in some diverse lines of safflower (Carthamus tinctorius L.) under salt stress. Pak. J. Bot., 39: 1937-1944.

Singh, R.K. and B.D. Chaudhary (1977). Biometrical methods in quantitative genetic analysis. New. Delhi, India: Kalyani Publishers.

Smith, H. and J. Jimmerson (2005). Safflower.This publication is a product of a collaborative research project between fort peck community college and the agricultural marketing policy center. Briefing No.58.

Tahernezhad, Z., J. Saba, M. Zeinalabedinll, S.S. Pourdad and M.R. Ghaffari (2018). Estamation of broad-sense heritability and variance components for seed yield and agronomic traits in native and exotic Safflower (Carthamus Tinctorius L.) Genotypes. Bangladesh J. Bot., 47 (3): 501 508.

Veena, K.R. and R.L. Ravikumar (2003). Mutagenic effect on homozygous parental lines heterozygous hybrids in altering character association in safflower. Karnataka J. Agric. Sci., 16: 390-396.

Velasco, L., B. Perez-Vich and J.M. FernandezMartinez (2005). Identification and genetic characterization of a safflower mutant with a modified tocopherol profile. Plant Breed., 124: 459-463

Weiss E.A. (1971). Safflower. In: Weiss EA (Ed) Castor Sesame and Safflower. Barnes and Noble, Inc, New York, 529-744.

Weiss, E.A. (2000). Safflower. In: Oil Seed Crops. $2^{\text {nd }}$ Ed. Blackwell Sci., Oxford, London, 364.

Yilmaz, H., M.B. Comak and F. Turgut (2016). Analysis of factors related to farmer's benefiting from safflower (Carthamus tinctorius L.,) production support: The case of central Anatolia in Turkey. J. Anim. Plant Sci., 26: 1411-1417.

Zhaomu, W. and D. Lijje (2001). Current situation and prospects of safflower products development in China. Proc. $5^{\text {th }}$ Int. safflower Conf., Jul.23.27,Williston, North Dakota and Sidney, MT, USA, 315-319. 
التقييم الور اثى لسلالات القرطم الطفرية مبكرة النضج وعديمة الأشثوالك للصفات الكمية ومحتوى الزيت

$$
\text { مروة السيد أحمد - سعيد سعد سليمان - ممدوح كامل أمين - محمد أبوبكر حسن يوسف }
$$

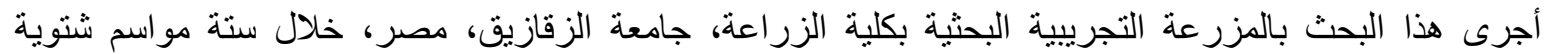

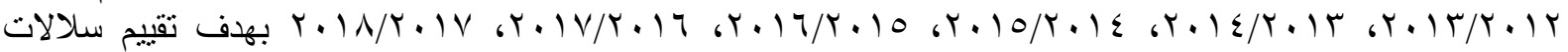

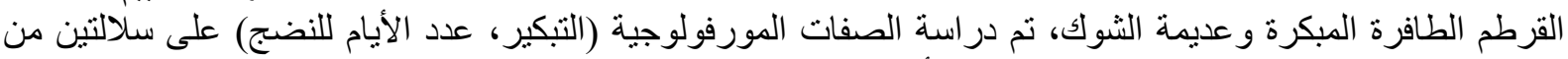

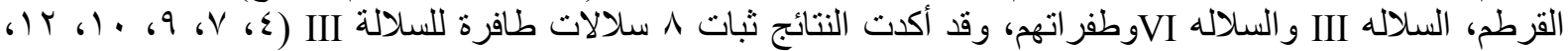

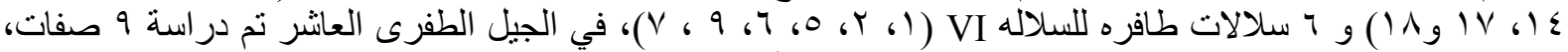

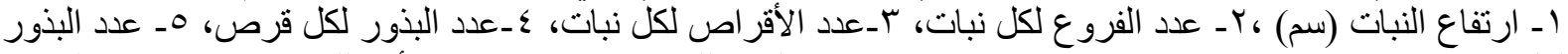

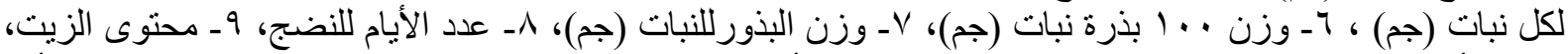

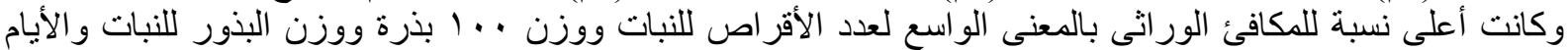

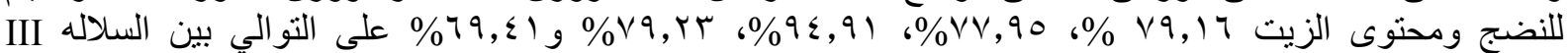

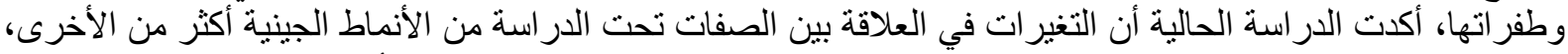

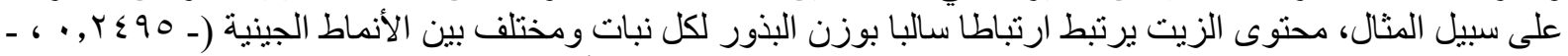

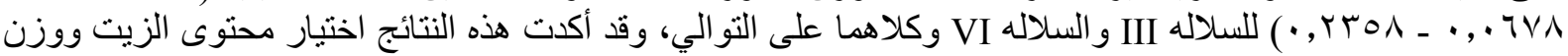

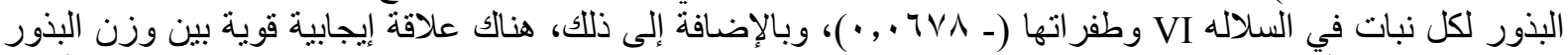

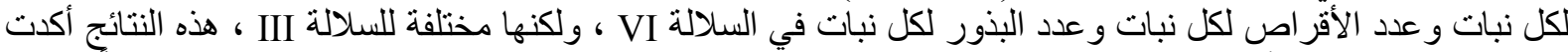

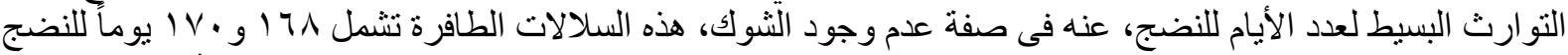

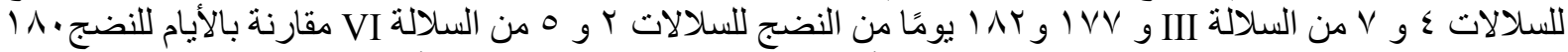

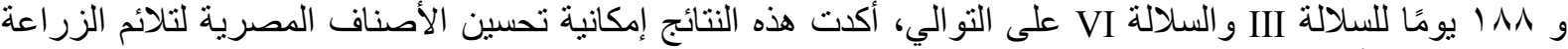
تحت ظروف الأر اضي الصحر اوية الفقيرة و القاسية. 